\title{
Elevada frecuencia de metilación del promotor de MLH1 mediada por sexo y edad en tumores colorrectales de pacientes mexicanos
}

José M. Moreno-Ortiz', Josselyn Jiménez-García1, Melva Gutiérrez-Angulo², Ma. de la Luz Ayala-Madrigal', Anahí González-Mercado", Christian O. González-Villaseñor', Beatriz A. Flores-López', Carlos Alvizo-Rodríguez", Jesús A. Hernández-Sandoval', Martha A. Fernández-Galindo', Víctor Maciel-Gutiérrez³, Helen Ramírez-Plascencia ${ }^{1}$ y Ruth Ramírez-Ramírez*

'Departamento de Biología Molecular y Genómica, Instituto de Genética Humana Dr. Enrique Corona Rivera, Centro Universitario de Ciencias de la Salud, Universidad de Guadalajara; ${ }^{2}$ Departamento de Ciencias de la Salud, Centro Universitario Los Altos, Universidad de Guadalajara; ${ }^{3}$ Servicio de Colon y Recto, Hospital Civil Dr. Juan I. Menchaca; ${ }^{4}$ Departamento de Biología Celular y Molecular, Centro Universitario de Ciencias Biológicas y Agropecuarias, Universidad de Guadalajara. Jalisco, México

\section{Resumen}

Introducción: Varios genes determinan el desarrollo de cáncer colorrectal (CCR), como MLH1, el cual codifica una proteína que participa en la reparación del ADN. La hipermetilación de MLH1 ha sido asociado con silenciamiento génico. Objetivo: Analizar la metilación de cinco regiones de la isla CpG de MLH1 en tumores colorrectales de pacientes mexicanos. Materiales y métodos: Se obtuvieron 101 muestras de tejido tumoral de pacientes mexicanos con CCR, quienes proporcionaron su consentimiento informado. El ADN fue sometido a conversión por bisulfito. La metilación de las cinco regiones de la isla CpG fue evaluada utilizando PCR específica para metilación. Resultados: La frecuencia de metilación en pacientes mexicanos con CCR fue del 25\%. La metilación de las regiones A y B fue el principal patrón observado (60\%). Las pacientes de sexo femenino mostraron una mayor frecuencia de metilación (71\%) (odds ratio: 3.085; intervalo de confianza; 1.85-8.03; $p=0.02) ; y$ del total de muestras metiladas, el $80 \%$ fueron individuos mayores de 45 años $(p<0.05)$. Conclusión: Calculamos una frecuencia de metilación para el gen MLH1 del 25\% en pacientes mexicanos con CCR, siendo el primer reporte para esta población. Pacientes de sexo femenino y pacientes mayores de 45 años mostraron una mayor frecuencia de metilación.

PALABRAS CLAVE: Cáncer colorrectal. Metilación. Población mexicana. MLH1.

\section{High frequency of MLH1 promoter methylation mediated by gender and age in colorectal tumors from Mexican patients}

\section{Abstract}

Background: Several genes determine the development of colorectal cancer (CRC), such as MLH1, which encodes a protein that participates in DNA repair. MLH1 hypermethylation has been associated with gene silencing. Objective: To analyze the methylation of five regions of MLH1 CPG island in colorectal tumors from Mexican patients. Material and methods: One hundred and one tumor tissue samples were obtained from Mexican patients with CRC who provided informed consent. DNA was subjected to bisulfite conversion. Methylation of all five regions of the $\mathrm{CpG}$ island was evaluated using methylation-specific PCR. Results: The frequency of methylation in Mexican patients with CRC was $25 \%$. Regions $A$ and B methylation was

Correspondencia:

*Ruth Ramírez-Ramírez

E-mail: ruth.ramirez@academicos.udg.mx
Fecha de recepción: 13-05-2021

Fecha de aceptación: 22-07-2021

DOI: $10.24875 / G M M .21000293$
Gac Med Mex. 2021;157:638-644

Disponible en PubMed

www.gacetamedicademexico.com

0016-3813/๑ 2021 Academia Nacional de Medicina de México, A.C. Publicado por Permanyer. Este es un artículo open access bajo la licencia CC BY-NC-ND (http://creativecommons.org/licenses/by-nc-nd/4.0/). 
the main observed pattern (60\%). Female patients showed a higher frequency of methylation (71\%) $(p=0.02, \mathrm{OR} 3.085, \mathrm{Cl}$ 1.85-8.03), and out of the of all the methylated samples $80 \%$ were from individuals older than 45 years $(p<0.05)$. Conclusion: We calculated a methylation frequency for the MLH1 gene of $25 \%$ in Mexican patients with CRC, with this being the first report for this population. Female patients and patients older than 45 years showed a higher frequency of methylation.

KEY WORDS: Colorectal cancer. Methylation. Mexican population. MLH1.

\section{Introducción}

El cáncer colorrectal (CCR) es la tercera causa de muerte relacionada con tumores en el mundo ${ }^{1,2}$. Aproximadamente el $45 \%$ de los casos ocurren en regiones subdesarrolladas ${ }^{1,3}$. EI CCR suele ocurrir en adultos con una edad promedio de 67 años $^{4}$. En México, en incidencia y mortalidad el CCR es el tercero más común en ambos sexos'.

El CCR es una neoplasia en la que ocurren procesos epigenéticos como la hipermetilación de islas CpG, lo que lleva a una inactivación de genes y a la alteración en el crecimiento y funcionamiento normal celular ${ }^{5}$. La hipermetilación de $M L H 1$ se ha descrito como un marcador pronóstico para esta neoplasia ${ }^{6}$. La proteína MLH1 forma parte de un complejo proteico que participa en el sistema de reparación por apareamiento erróneo ${ }^{7-9}$. El gen MLH1 se localiza en 3p22.2 y contiene 19 exones $^{10}$. Su expresión está regulada vía promotor, carece de caja TATA, pero contiene la secuencia CCAAT necesaria para la unión de factores de transcripción ${ }^{11}$. MLH1 tiene una isla CpG de 1128 pb, en la que se encuentran 93 sitios CpG (chr3: 3703422937035356) ${ }^{12}$. En CCR esporádico, MLH1 está metilado del 10 al 15\% $\%^{5}$. En líneas celulares de carcinoma colorrectal han sido estudiadas cuatro regiones de la isla CpG: A (-711 a -577), B (-522 a -266), C (-248 a -178$)$ y $D(-109 a+5)$, correlacionando hipermetilación con inhibición de la expresión génica de $M L H 1^{13,14}$. Por otro lado, en población japonesa se ha reportado el análisis de cinco regiones: A (-755 a -574), B (-597 a -393), C (-420 a -188), D (-286 a -53) y $E(-73 a+86)$, correlacionando la metilación total y parcial con las características clínico-patológicas, expresión proteica e inestabilidad de microsatélites ${ }^{15}$.

Específicamente, no existe ningún reporte de metilación de MLH1 en pacientes mexicanos con CCR. Debido a esto, el propósito del presente estudio fue analizar la frecuencia de metilación de cinco regiones de la isla CpG de MLH1 en pacientes mexicanos con CCR.

\section{Materiales y métodos}

\section{Muestras}

Se colectaron 101 muestras de tejido tumoral de pacientes mexicanos diagnosticados clínica e histopatológicamente con CCR en el Hospital Civil Dr. Juan I. Menchaca (Guadalajara, Jalisco, México). Las muestras de tejido se obtuvieron al término de la cirugía. Todos los pacientes firmaron un consentimiento informado con base en la Declaración de Helsinki. Este estudio fue aprobado por el comité local de bioética (Cl-01417).

\section{Extracción de ADN y cuantificación}

Se realizó la extracción de ADN de los tejidos tumorales (High Pure PCR Template Preparation kit [n. de producto: 11796828001], Roche Diagnostic $\mathrm{GmbH}$, Mannheim, Alemania). Posteriormente se cuantificó el DNA por espectrofotometría. Las muestras se almacenaron a $-20{ }^{\circ} \mathrm{C}$ hasta su uso.

\section{Conversión de ADN por bisulfito}

Se utilizó una concentración de ADN de $100 \mu \mathrm{g} / \mathrm{ml}$ $(18 \mu \mathrm{l})$ y posteriormente se trató con el kit EZ DNA Methylation-Gold ( $\mathrm{n}^{\circ}$ de producto: D5006; ZYMO Research, EE.UU.). Se utilizó como control ADN de la línea celular HCT116 DKO (n. ${ }^{\circ}$ de producto: D5014; ZYMO Research) la cual se usó durante el análisis de conversión y reacción en cadena de la polimerasa específica para metilación (MS-PCR).

\section{Reacción en cadena de la polimerasa específica para metilación}

La isla CpG se dividió en cinco regiones para el análisis de metilación: A (-539 a -677), B (-418 a -264), C (-162 a -62), D (+82 a +260) y E (+234 a +415). Se realizó MS-PCR para todas las regiones utilizando ADN convertido. Los iniciadores utilizados para ADN metilado y no metilado se describen en la tabla 1. La 
Tabla 1. Iniciadores del gen $M L H 1$ utilizados para la amplificación del ADN metilado y no metilado de las regiones A, B, C, D, y E

\begin{tabular}{|c|c|c|c|}
\hline $\begin{array}{l}\text { Regiones } \\
\text { de la isla CpG }\end{array}$ & M/U & iniciadores & Tamaño del fragmento (pb) \\
\hline \multirow[t]{4}{*}{ A } & \multirow[t]{2}{*}{ M } & F5' -CGGTAGAGTTCGAGGTTTGTAC -3' & \multirow[t]{2}{*}{134} \\
\hline & & R5' -CACGAATACTACGAACGATATATAACG -3' & \\
\hline & \multirow[t]{2}{*}{$U$} & F5' GTGGTAGAGTTTGAGGTTTGTATGA -3' & \multirow[t]{2}{*}{138} \\
\hline & & R5' -AAACACAAATACTACAAACAATATATAACA -3' & \\
\hline \multirow[t]{4}{*}{ B } & \multirow[t]{2}{*}{ M } & F5' - GTCGGAAAATTAGAGTTTCGTC -3' & \multirow[t]{2}{*}{151} \\
\hline & & R5' -GCAAAACGAAAAAAATACTTAACG -3' & \\
\hline & \multirow[t]{2}{*}{$U$} & F5' - GGTTGGAAAATTAGAGTTTTGTTGA -3' & \multirow[t]{2}{*}{154} \\
\hline & & R5' -ACAAAACAAAAAAAATACTTAACACA -3' & \\
\hline \multirow[t]{4}{*}{ C } & \multirow[t]{2}{*}{ M } & F5' -GATAGCGATTTTTAACGC -3' & \multirow[t]{2}{*}{93} \\
\hline & & R5' - TCTATAAATTACTAAATCTCTTCG -3' & \\
\hline & \multirow[t]{2}{*}{$U$} & F5' -AGAGTGGATAGTGATTTTTAATGT -3' & \multirow[t]{2}{*}{100} \\
\hline & & R5' -ACTCTATAAATTACTAAATCTCTTCA -3' & \\
\hline \multirow[t]{4}{*}{ D } & \multirow[t]{2}{*}{ M } & F5' -GTTTTTTTGGCGTTAAAATGTC -3' & \multirow[t]{2}{*}{166} \\
\hline & & R5' - CCTTAAATAAACCCGACTCGAC -3' & \\
\hline & \multirow[t]{2}{*}{$U$} & F5' -TTGGTTTTTTTGGTGTTAAAATGTT -3' & \multirow[t]{2}{*}{172} \\
\hline & & R5' -AACCCTTAAATAAACCCAACTCAAC -3' & \\
\hline \multirow[t]{4}{*}{ E } & \multirow[t]{2}{*}{ M } & F5' -GAGTCGGGTTTATTTAAGGGTTAC -3' & \multirow[t]{2}{*}{177} \\
\hline & & R5' -GATAAAAAAACACACGATCTACGAA -3' & \\
\hline & \multirow[t]{2}{*}{$U$} & F5' -AGTTGGGTTTATTTAAGGGTTATGA -3' & \multirow[t]{2}{*}{176} \\
\hline & & R5' - AATAAAAAAACACACAATCTACAAA -3' & \\
\hline
\end{tabular}

MS-PCR para todas las regiones se realizó a 30 ciclos, en un volumen de $12 \mu \mathrm{l}$, el cual contenía $100 \mathrm{ng} / \mu \mathrm{l}$ DNA, $1 \times$ buffer $(500 \mathrm{mM} \mathrm{KCl}, 100 \mathrm{mM}$ Tris$\mathrm{HCl}$ y $0.1 \%$ Triton $\left.^{\mathrm{TM}} \mathrm{X}-100\right), 1.5 \mathrm{mM} \mathrm{MgCl}, 2 \mathrm{mM}$ dNTP, $10 \mathrm{pM}$ de cada iniciador y $2 \mathrm{U}$ de Taq DNA polimerasa Platinum. La desnaturalización inicial se llevó a $95^{\circ} \mathrm{C}$ por $5 \mathrm{~min}$, seguido de $94^{\circ} \mathrm{C}$ por $45 \mathrm{~s}$, alineamiento a $57{ }^{\circ} \mathrm{C}$ por $45 \mathrm{~s}$ y elongación a $72{ }^{\circ} \mathrm{C}$ por $1 \mathrm{~min}$. Los productos de PCR se visualizaron en geles de poliacrilamida teñidos con nitrato de plata al $6 \%$.

\section{Análisis estadístico}

Para el análisis estadístico se usó el software SPSS v25 (SPSS, Inc., Chicago, IL, EE.UU.). Las pruebas exacta de Fisher, $\chi^{2} y$ odds ratio (OR), fueron utilizadas para el análisis de metilación. Se consideraron significativos valores de $p<0.05$.

\section{Resultados}

Se analizaron 101 muestras de tejido tumoral de pacientes mexicanos con CCR, la edad promedio fue de 59 años. Las características analizadas fueron sexo (52\% hombres), actividad física (53\%), diabetes mellitus tipo II (21\%), consumo de tabaco, alcohol, carne roja, frutas y verduras $(42,49,81$ y $75 \%$, respectivamente), metástasis (42\%), presencia de pólipos $(17 \%)$, recaídas $(4 \%)$ y localización del tumor (colon, 60\%).

\section{Análisis de metilación}

La frecuencia de metilación de MLH1 en pacientes mexicanos con CCR fue del $25 \%$. Sin embargo, del total de muestras metiladas, la frecuencia de metilación fue del $60 \%$ en regiones A y B (Tabla 2). 
Tabla 2. Análisis de metilación de la regiones de la isla CpG en el gen $M L H 1$ de tejido tumoral de pacientes con cáncer colorrectal

\begin{tabular}{|l|c|}
\multirow{2}{*}{ Región metilada } & Total de muestras metiladas \\
\cline { 2 - 2 } & $\mathrm{n}=\mathbf{2 5}(\%)$ \\
\hline A & $15(60)$ \\
\hline B & $15(60)$ \\
\hline C & $7(28)$ \\
\hline D & $8(32)$ \\
\hline E & $7(28)$ \\
\hline
\end{tabular}

Se comparó la frecuencia de metilación con las características clínico-patológicas y de estilo de vida, encontrándose diferencias significativas con el sexo y la edad ( $p<0.05)$ (Tabla 3). Del total de muestras metiladas, el $68 \%$ correspondieron a pacientes de sexo femenino. Además, se establecieron dos rangos de edad (< 45 y > 45 años de edad); del total de muestras metiladas, el $80 \%$ presentó metilación a una edad mayor de 45 años.

Se comparó la frecuencia de metilación (25\%) con las frecuencias reportadas en otras poblaciones, encontrándose diferencias significativas (Tabla 4).

\section{Discusión}

\section{Análisis de metilación}

En el presente estudio se analizaron cinco regiones de la isla $C p G$. Cabe mencionar que las regiones $D$ y $\mathrm{E}$ no habían sido previamente analizadas por otros autores (Tabla 5). Las cinco regiones analizadas cubren un total de 63 sitios de la isla CpG.

Estudios previos analizaron cuatro regiones ricas en sitios CpG mediante secuenciación; sin embargo, fueron utilizadas líneas celulares, cubriendo 43 sitios CpG. Particularmente, estos estudios mostraron una correlación entre la metilación de la región $\mathrm{C}$ o D con la inhibición de la expresión génica debido a la presencia de la secuencia CCAAT, la metilación de esta secuencia inhibe la unión del factor de transcripción CBF (core binding factor). De acuerdo con estos estudios, la hipermetilación en las regiones $C$ y $D$ del promotor MLH1 provoca la inactivación y deficiencia de la proteína ${ }^{13,14}$. En nuestro estudio, el $28 \%$ de las muestras se encontraron metiladas en la región $\mathrm{C}$ y el $32 \%$ metiladas en la región $\mathrm{D}$. Estas regiones se encuentran alrededor del sitio de inicio de la transcripción, pero siempre tienen una frecuencia de metilación más baja que las otras regiones ${ }^{22}$. Por ejemplo, las regiones $\mathrm{A}$ y $\mathrm{B}$ son las más frecuentemente metiladas $^{23}$, sin embargo, el efecto funcional de estas regiones no se ha descrito directamente. Al respecto, en el presente estudio se observó una mayor frecuencia de metilación en las regiones A y B $(60 \%)$. Otro reporte reveló que la región $A$ puede ser el primer sitio que se metile durante la progresión a un estado de metilación ${ }^{15}$. Estas regiones $(A, B)$ son las más alejadas del inicio del sitio de transcripción. Por otro lado, todos los tumores metilados en la región $C$ mostraron metilación en las regiones $\mathrm{A}$ y $\mathrm{B}$, lo cual fue una constante en nuestro estudio, pero los autores mostraron casos de metilación solo en las regiones $A$ y $B, y$ no en la región $\mathrm{C}^{13}$.

La frecuencia de la metilación fue del $25 \%$ para MLH1. Se conoce que la metilación del ADN puede afectar sitios de unión de factores de transcripción, elementos reguladores y la conformación de la cromatina, lo que da como resultado múltiples niveles de control de la expresión ${ }^{24}$. Esta frecuencia es mayor que la propuesta por otros autores, quienes mencionan una frecuencia de metilación para $M L H 1$ entre el $10-15 \%$ en casos de CCR esporádico ${ }^{5}$. Incluso nuestro porcentaje también fue mayor que el reportado en otras poblaciones ${ }^{7,16-21}$.

\section{Metilación mediada por sexo}

El CCR es una enfermedad compleja que involucra varios factores ${ }^{25-28}$. En el presente estudio se examinó la correlación de algunos de estos con la metilación. Sin embargo, solo se presentaron diferencias significativas con sexo y edad. Específicamente, la metilación de MLH1 se ha asociado con variables clínico-patológicas, incluido el sexo femenino ${ }^{29}$. En el presente estudio, la frecuencia de metilación fue mayor en mujeres (OR: 3.085; intervalo de confianza [IC: 1.85-8.03; $p=0.02$ ); estos hallazgos son similares a los reportados en diferentes estudios ${ }^{16,18}$. Un reporte que incluyó 210 muestras de pacientes con CCR reveló una metilación más elevada en las mujeres $(p=0.007)$. Además, se propuso que la presencia de la variante $M L H 1$ c. $-93 G>A$ (rs1800734) conduce a un mayor riesgo de metilación en mujeres ${ }^{21}$. Es interesante mencionar que en este trabajo las mujeres que tuvieron alguna región metilada tuvieron en común la presencia de sobrepeso u obesidad, y en alguna etapa de su vida habían consumido tabaco y alcohol. 
Tabla 3. Comparación en la frecuencia de metilación de las regiones de la islas CpG en el gen MLH1 por sexo

\begin{tabular}{|c|c|c|c|c|c|c|}
\hline \multirow[t]{3}{*}{ Regiones } & \multicolumn{4}{|c|}{ Pacientes CCR $(n=101)$} & \multirow[t]{3}{*}{ p } & \multirow[t]{3}{*}{ OR (IC) } \\
\hline & \multicolumn{2}{|c|}{ Sexo masculino $(n=53)$} & \multicolumn{2}{|c|}{ Sexo femenino $(n=48)$} & & \\
\hline & M & U & M & U & & \\
\hline Cualquier región* & $8(15.1 \%)$ & $45(84.9 \%)$ & $17(35.4 \%)$ & $31(64.6 \%)$ & 0.018 & $3.085(1.9-8.0)$ \\
\hline A & $3(5.7 \%)$ & $50(94.3 \%)$ & $12(25.0 \%)$ & $36(75.0 \%)$ & 0.01 & $5.556(1.5-21.1)$ \\
\hline B & $4(7.5 \%)$ & $49(92.5 \%)$ & $11(22.9 \%)$ & $37(77.1 \%)$ & 0.048 & $3.642(1.1-12.4)$ \\
\hline$A+B$ & $2(3.8 \%)$ & $51(96.2 \%)$ & $9(18.8 \%)$ & $39(81.3 \%)$ & 0.023 & $5.885(1.2-28.8)$ \\
\hline
\end{tabular}

*Muestras metiladas en cualquier región analizada (A, B, C, D, E).

CCR: cáncer colorrectal; M: metilado; OR: odds ratio; IC: intervalo de confianza; U: no metilado.

Tabla 4. Frecuencia de metilación del gen $M L H 1$ entre diferentes poblaciones de pacientes con cáncer colorrectal

\begin{tabular}{|c|c|c|c|c|c|}
\hline Población & $\mathrm{n}$ & Tejido tumoral analizado & $\mathrm{M}(\%)$ & p & Referencia \\
\hline Australiana & 946 & Tejido fresco & 10.1 & 0.0001 & Wong, et al., $2011^{16}$ \\
\hline Japonesa & 104 & Tejido fresco & 9.6 & 0.0039 & Hokazono, et al., $2014^{17}$ \\
\hline Japonesa & 210 & Tejido fresco & 28.5 & 0.4935 & Miyakura, et al., $2014^{18}$ \\
\hline China & 301 & Tejido fresco & 14.6 & 0.0188 & Wang, et al., $2014^{19}$ \\
\hline Española & 326 & Tejido fresco & 5.2 & 0.0001 & Veganzones, et al., $2015^{20}$ \\
\hline Coreana & 132 & Tejido fresco & 6 & 0.0001 & Lee, et al., $2019^{21}$ \\
\hline Eslovaca & 300 & Tejido parafinado & 7.6 & 0.0001 & Kašubová, et al., $2019^{7}$ \\
\hline Mexicana & 101 & Tejido fresco & 25 & - & Presente estudio \\
\hline
\end{tabular}

M: porcentaje de alelos metilados.

Tabla 5. Comparación entre diferentes regiones de la isla CpG del gen MLH1 reportado por Deng, et al. (2001), Miyakura, et al. (2014) y el presente estudio

\begin{tabular}{|l|c|c|c|}
\hline Región & $\begin{array}{c}\text { Deng, et al., } \\
2001^{14}\end{array}$ & $\begin{array}{c}\text { Miyakura, et al., 2001' } \\
\text { Miyakura, et al., 2014 }\end{array}$ & Este estudio \\
\hline A & $-711 a-577$ & $-755 a-574$ & $-677 a-539$ \\
\hline B & $-552 a-266$ & $-597 a-393$ & $-418 a-264$ \\
\hline C & $-248 a-178$ & $-420 a-188$ & $-162 a-62$ \\
\hline D & $-109 a+5$ & $-286 a-53$ & $+82 a+260$ \\
\hline E & & $-73 a+86$ & $+234 a+415$ \\
\hline
\end{tabular}

Al respecto existe suficiente evidencia de la influencia de estos factores en la metilación del ADN ${ }^{30-32}$.

\section{Metilación mediada por la edad}

Asimismo, el análisis de la frecuencia de metilación por rango de edad reveló una mayor metilación en individuos mayores de 45 años (80\%). Sin embargo, el efecto de la edad sobre el estado de metilación sigue sin ser claro. El único estudio en MLH1 que asoció el envejecimiento con la metilación aberrante mostró que la metilación aumentó con la edad (frecuencia por 10 años de edad $=2.1 \% ; p<0.001)^{33}$. Además, se encontró que la metilación de MLH1 en muestras de tumor ocurrieron con mayor frecuencia en mayores de 50 años $^{29}$. En nuestro estudio, los niveles elevados de metilación fueron más frecuentes en mujeres mayores de 45 años.

\section{Análisis poblacional de metilación}

El análisis poblacional mostró que la frecuencia de metilación en pacientes con CCR en población mexicana fue significativamente diferente a otras poblaciones: australiana, japonesa, china, española, coreana y eslovaca ${ }^{7,16-21}$. Estas diferencias probablemente se deben a la variabilidad en el número de muestras 
utilizadas en cada estudio, las diferentes regiones analizadas por cada autor, el estilo de vida y la estructura genética característica de los mexicanos. Sin embargo, en el presente estudio no se encontraron datos significativos cuando se analizó la asociación de la metilación con aspectos relacionados con el estilo de vida.

\section{Conclusión}

El presente análisis mostró una frecuencia de metilación de $M L H 1$ del $25 \%$. Este valor es superior al reportado en otras poblaciones y constituye el primer reporte en pacientes mexicanos con CCR. El análisis por región reveló que las regiones $\mathrm{A}$ y $\mathrm{B}$ tuvieron una mayor frecuencia de metilación (60\%). Finalmente, el $80 \%$ de las muestras metiladas correspondieron a pacientes mayores de 45 años y se encontró una elevada metilación en pacientes del sexo femenino, lo que nos indica un papel relevante de estos dos factores en la metilación del ADN.

\section{Agradecimientos}

El estudio fue financiado por PRODEP. Christian Octavio González-Villaseñor, Beatriz Armida LópezFlores, Carlos Alvizo-Rodríguez, Jesús Arturo Hernández-Sandoval, Martha Alejandra Fernández Galindo y Helen Ramírez-Plascencia recibieron beca doctoral por parte del CONACyT.

\section{Financiamiento}

EI estudio fue financiado por PRODEP
UDG-PTC-1456.

\section{Conflicto de intereses}

Los autores declaran no tener ningún conflicto de intereses.

\section{Responsabilidades éticas}

Protección de sujetos humanos y animales. Los autores declaran que los procedimientos seguidos fueron de acuerdo con las regulaciones del comité de ética de investigación y con el Código de ética de la Asociación Médica Mundial (Declaración de Helsinki).

Confidencialidad de los datos. Los autores declaran haber seguido los protocolos de su centro de trabajo sobre la publicación de datos de pacientes.
Derecho a la privacidad y consentimiento informado. Los autores han obtenido el consentimiento informado por escrito de los pacientes o sujetos mencionados en el artículo. El autor correspondiente está en posesión de este documento.

\section{Bibliografía}

1. Bray F, Ferlay J, Soerjomataram I, Siegel RL, Torre LA, Jemal A. Global Cancer Statistics 2018: GLOBOCAN Estimates of Incidence and Mortality Worldwide for 36 Cancers in 185 Countries. CA Cancer J Clin. 2018;68:394-424.

2. Han S, Gao J, Zhou Q, Liu S, Wen S, Yang X. Role of intestinal flora in colorectal cancer from the metabolite perspective: a systematic review. Cancer Manag Res. 2018;31:199-206.

3. Aran V, Victorino AP, Thuler LC, Ferreira CG. Colorectal cancer: Epidemiology, disease mechanisms and interventions to reduce onset and mortality. Clin Colorectal Cancer. 2016;15:195-203.

4. Weinberg BA, Marshall JL. Colon cancer in young adults: Trends and their implications. Curr Oncol Rep. 2019;21:3.

5. Tse JW, Jenkins LJ, Chionh F, Mariadason JM. Aberrant DNA methylation in colorectal cancer: What should we target? Trends Cancer. 2017;3:698-712.

6. Xicola RM, Llor X. DNA methylation defects in sporadic and hereditary colorectal cancer. Gastroenterol Hepatol. 2012;35:480-7.

7. Kašubová I, Kalman M, Jašek K, Burjanivová $T$, Malicherová $B$, Vaňochová A, et al. Stratification of patients with colorectal cancer without the recorded family history. Oncol Lett. 2019;17:3649-56.

8. Sacho EJ, Kadyrov FA, Modrich PTA, Erie DA. Direct visualization of asymmetric adenine nucleotide-induced conformational changes in MutLo. Mol Cel. 2008;29:1-20.

9. Kobayashi H, Ohno S, Sasaki Y, Matsuura M. Hereditary breast and ovarian cancer susceptibility genes. Oncol Rep. 2013;30:1019-29.

10. Baert-Desurmont S, Buisine MP, Bessenay E, Frerot S, Lovecchio T, Martin $\mathrm{C}$, et al. Partial duplications of the MSH2 and MLH1 genes in hereditary nonpolyposis colorectal cancer. Eur J Hum Genet. 2007;15:383-6.

11. Ito $E$, Yanagisawa $Y$, Iwahashi $Y$, Suzuki $Y$, Nagasaki $H$, Akiyama $Y$, et al. A core promoter and a frequent single-nucleotide polymorphism of the mismatch repair gene hMLH1. Biochem Biophys Res Commun. 1999;256:488-94.

12. Kent WJ, Sugnet CW, Furey TS, Roskin KM, Pringle TH, Zahler AM, et al. The human genome browser at UCSC. Genome Res. 2002:12:996-1006.

13. Deng G, Chen A, Hong J, Chae HS, Kim YS. Methylation of CpG in a small region of the $\mathrm{hMLH} 1$ promoter invariably correlates with the absence of gene expression. Cancer Res. 1999;59:2029-33.

14. Deng G, Chen A, Pong E, Kim YS. Methylation in hMLH1 promoter interferes with its binding to transcription factor CBF and inhibits gene expression. Oncogene. 2001;20:7120-7.

15. Miyakura Y, Sugano K, Konishi F, Ichikawa A, Maekawa M, Shitoh K, et al. Extensive methylation of hMLH1 promoter region predominates in proximal colon cancer with microsatellite instability. Gastroenterology. 2001;121:1300-9

16. Wong JJ, Hawkins NJ, Ward RL, Hitchins MP. Methylation of the 3p22 region encompassing $\mathrm{MLH} 1$ is representative of the $\mathrm{CpG}$ island methylator phenotype in colorectal cancer. Mod Pathol. 2011;24:396-411.

17. Hokazono K, Ueki T, Nagayoshi K, Nishioka Y, Hatae T, Koga Y, et al. A CpG island methylator phenotype of colorectal cancer that is contiguous with conventional adenomas, but not serrated polyps. Oncol Lett. 2014:8:1937-44

18. Miyakura Y, Tahara M, Lefor AT, Yasuda Y, Sugano K. Haplotype defined by the MLH1-93G/A polymorphism is associated with $M L H 1$ promoter hypermethylation in sporadic colorectal cancers. BMC Res Notes. 2014;7:1-11.

19. Wang Y, Li D, Li X, Teng C, Zhu L, Cui B, et al. Prognostic significance of $h M L H 1 / h M S H 2$ gene mutations and $h M L H 1$ promoter methylation in sporadic colorectal cancer. Med Oncol. 2014;31:1-8.

20. Veganzones S, Maestro ML, Rafael S, de la Orden V, Vidaurreta M, Mediero B, et al. Combined methylation of p16 and hMLH1 (CMETH2) discriminates a subpopulation with better prognosis in colorectal cancer patients with microsatellite instability tumors. Tumor Biol. 2015;36:3853-61.

21. Lee JA, Park HE, Yoo SY, Matsuura M. CpG island methylation in sessile serrated adenoma/polyp of the colorectum: Implications for differential diagnosis of molecularly high-risk lesions among non-dysplastic sessile serrated adenomas/polyps. J Pathol Transl Med. 2019;53:225-35.

22. Van IJ, Niessen HE, Derks S, Baldewijns MM, van Criekinge W, Herman JG, et al. Analysis of promoter CpG island hypermethylation in cancer. Location, location, and location! Clin Cancer Res. 2011;17:4225-31. 
23. Li X, Yao X, Wang $Y$, Hu F, Wang F, Jiang L, et al. MLH1 promote methylation frequency in colorectal cancer patients and related clinicopathological and molecular features. PLoS One. 2013;8:e59064.

24. Jones MJ, Goodman SJ, Kobor MS. DNA methylation and healthy human aging. Aging Cell. 2015;14:924-32.

25. Derry MM, Raina KC, Agarwal R. Identifying molecular targets of lifestyle modifications in colon cancer prevention. Front Oncol. 2013;3:1-20.

26. Jung SE, Shin KJ, Lee HY. DNA methylation-based age prediction from various tissues and body fluids. BMB Rep. 2017;50:546-53.

27. Hadjipetrou A, Anyfantakis D, Galanakis CG, Kastanakis M, Kastanakis S. Colorectal cancer, screening and primary care: A mini literature review. World J Gastroenterol. 2017;23:6049-58.

28. Dolatkhah R, Somi MH, Shabanloei R, Farassati F, Fakhari A, Dastgiri S Main risk factors association with proto-oncogene mutations in colorectal cancer. Asian Pac J Cancer Prev. 2018;19:2183-90.
29. Levine AJ, Phipps Al, Baron JA, Buchanan DD, Ahnen DJ, Cohen SA, et al. Clinicopathologic risk factor distributions for MLH1 promoter region methylation in CIMP-positive tumors. Cancer Epidemiol Biomarkers Prev. 2016;25:68-75.

30. Varela-Rey M, Woodhoo A, Martinez-Chantar ML, Mato JM, Lu SC. Alcohol, DNA methylation, and cancer. Alcohol Res. 2013;35:25-35.

31. Jin F, Thaiparambil J, Donepudi SR, Vantaku V, Piyarathna DWB, Maity S, et al. Tobacco-specific carcinogens induce hypermethylation, DNA adducts, and DNA damage in bladder cancer. Cancer Prev Res (Phila). 2017; 10:588-97.

32. Parrillo L, Spinelli R, Nicolò A, Longo M, Mirra P, Raciti GA, et al. Nutritional factors, DNA methylation, and risk of type 2 diabetes and obesity: Perspectives and challenges. Int J Mol Sci. 2019;20:2983.

33. Noreen F, Röösli M, Gaj P, Pietrzak J, Weis S, Urfer P, et al. Modulation of age- and cancer-associated DNA methylation change in the healthy colon by aspirin and lifestyle. J Natl Cancer Inst. 2014;106:dju161. 